\title{
Comparative Pulsation Calculations with OP and OPAL Opacities
}

\author{
S.M. Kanbur ${ }^{1}$, N.R. Simon ${ }^{1}$ \\ ${ }^{1}$ Department of Physics and Astronomy, University of Nebraska-Lincoln, NE \\ 68588-0111
}

The OPAL opacities (Iglesias and Rogers 1991; Iglesias, Rogers and Wilson 1992) are recent revisions to the traditional Los Alamos (LA) opacities and have proven very successful in resolving the Cepheid beat mass, and to a lesser extent, the Cepheid bump mass discrepancies (Moskalik, Buchler and Marom 1991, hereafter MBM). MBM showed that for the beat Cepheids the P1/P0 period ratios, calculated with OPAL, yielded solar masses between 4 and 7 , in agreement with other mass determinations. MBM also found that the P2/P0 period ratios were reduced, implying higher bump Cepheid masses, though the conflict with standard evolutionary masses was not completely eliminated. We have made linear nonadiabatic pulsation calculations with another set of recently computed opacities, namely those of the Opacity Project (Seaton 1987, 1992, hereafter OP). Since the two teams have employed different techniques in their calculations, it is of interest to compare pulsation results using the two sets of opacities.

Our conclusion is that the OPAL and OP opacities cannot be differentiated on the basis of our calculations. The Becker Iben Tuggle (1977) mass luminosity relation is sufficient for the beat Cepheids, but has too high a period ratio (by 0.01) at a fundamental period of 10 days for bump Cepheids. The mass luminosity relation involving substantial core overshoot (Chiosi 1988) can model both beat and bump Cepheids, but with lower (by about a solar mass) masses.

\section{References:}

Becker, S.A., Iben, I. \& Tuggle, R.S., 1977, Astrophys. J., 218, 633.

Chiosi, C., 1989 in 'The Use of Pulsating Stars in Fundamental Problems of Astronomy', Cambridge University Press, $p 19$.

Iglesias, C.A., \& Rogers, F.J., 1991, Astrophys. J., 371, 408.

Iglesias, C.A., Rogers, F.J., \& Wilson, B. 1992, preprint.

Moskalik, P., Buchler, J. R., \& Marom, A., 1992, Astrophys. J., 385, 685 (MBM).

Seaton, M.J., 1987, J. Phys. B., 20, 6363.

Seaton, M.J., private communication. 